\title{
An equilibrium closure result for discontinuous games
}

\author{
Erik J. Balder
}

Received: 17 September 2009 / Accepted: 3 October 2010 / Published online: 21 October 2010 (C) The Author(s) 2010. This article is published with open access at Springerlink.com

\begin{abstract}
For games with discontinuous payoffs Simon and Zame (Econometrica 58:861-872, 1990) introduced payoff indeterminacy, in the form of endogenous sharing rules, which are measurable selections of a certain payoff correspondence. Their main result concerns the existence of a mixed Nash equilibrium and an associated sharing rule. Its proof is based on a discrete approximation scheme "from within" the payoff correspondence. Here, we present a new, related closure result for games with possibly noncompact action spaces, involving a sequence of Nash equilibria. In contrast to Simon and Zame (Econometrica 58:861-872, 1990), this result can be used for more involved forms of approximation, because it contains more information about the endogenous sharing rule. With such added precision, the closure result can be used for the actual computation of endogenous sharing rules in games with discontinuous payoffs by means of successive continuous interpolations in an approximation scheme. This is demonstrated for a Bertrand type duopoly game and for a location game already considered by Simon and Zame. Moreover, the main existence result of Simon and Zame (Econometrica 58:861-872, 1990) follows in two different ways from the closure result.
\end{abstract}

Keywords Nash equilibrium - Discontinuous games - Weak convergence of probability measures $\cdot$ Endogenous sharing rule $\cdot$ Kuratowski limes superior

JEL Classification C72

A question by an anonymous referee helped and stimulated the author to demarcate the present paper's position with respect to Simon and Zame (1990). Also, remarks by Guilherme Carmona helped to improve the presentation.

E. J. Balder ( $\varangle)$

Mathematical Institute, University of Utrecht, P.O. Box 80.010, 3508 TA Utrecht, The Netherlands e-mail: E.J.Balder@uu.nl 


\section{Introduction}

Discontinuous games, with their associated problems regarding the formulation and existence of appropriate equilibrium notions, form a subject of long-standing interest in economics. The oldest of these, Bertrand's duopoly game, dates back to 1883. Such games are characterized by the fact that they have discontinuities in their payoffs as a function of player's actions. For instance, in Bertrand's duopoly game the firm which sets the lowest price captures the entire market. Several other examples of discontinuous games can be found in Dasgupta and Maskin (1986a,b). In the face of such discontinuity, general theorems about the existence of Nash equilibria for such games would seem to be out of the question. Nevertheless, quite some progress has been made in this direction. Let us recall in this connection the already mentioned papers by Dasgupta and Maskin (1986a,b) and the papers by Simon (1987), Lebrun (1996) and Reny (1999). The former two papers suppose a particular structure of the payoff discontinuities; this was refined by Simon (1987). These papers use an approximation by finite games and their associated mixed Nash equilibria. In a far-reaching generalization of this work, Reny (1999) showed that the question of finding Nash equilibria can be transformed by means of his better-reply security condition. In this way, it is possible to find pure equilibrium existence results as well (this contrasts notably with the other cited papers). In a different direction, Simon and Zame (1990) introduced a new approach to the existence of mixed equilibria, based on an upper semicontinuous payoff correspondence with compact convex values (if the initial payoff function is discontinuous, then they use the smallest upper semicontinuous and convex-compactvalued correspondence which has that payoff as its selection). The equilibrium payoff function, which they call endogenous sharing rule but which we prefer to call by the more neutral name of resolvent payoff profile, is required to be a measurable selection of this correspondence. Thus, Simon and Zame restore continuity of the payoff structure at the price of payoff indeterminacy. As observed by Jackson et al. (2002), the precise connections between Simon and Zame (1990) and the developments started by Reny (1999) are as yet not well understood.

The present paper continues the analysis started in Simon and Zame (1990). First, we consider a sequence of games and associated mixed Nash equilibria, for which we formulate a closure result. It addresses the following question: if the sequence of mixed Nash equilibrium profiles converges weakly to a mixed action profile, then is the latter also a Nash equilibrium for a payoff profile that is closely related to the payoffs in the sequence? Theorem 1 provides the answer to this question. Unlike Simon and Zame (1990), where it was enough for payoffs to be measurable selections of the payoff correspondence, it makes precise what has to be understood by "closely" in the preceding question. Notably, this opens the way to interpolation schemes whereby one interpolates continuously over the points of discontinuity of an original payoff profile. More precisely, for a sequence of payoff profiles, as considered here and in the Corollary of Simon and Zame (1990), the approach followed by Simon and Zame works with the smallest payoff correspondence which is convex-compact-valued, upper semicontinuous and contains all the graphs of the payoff profiles in the sequence. For interpolation schemes as described above, such a payoff correspondence would simply be too large. In contrast, this paper shows that a smaller version of the payoff correspondence 
can be used, replacing Simon and Zame's use of closures by Kuratowski limes superior sets (compare formulas (6) and (9) below). Because of its inherent limit properties, this smaller payoff correspondence can be used for interpolation arguments of the type mentioned above. Apart from this major point of difference with Simon and Zame (1990), another one is that our closure result works with possibly noncompact action spaces. To reveal some more details, the key contribution of the closure result in Theorem 1 is formed by its limit properties (6)-(7) for the endogenous sharing rule/resolvent payoff profile (i.e., the payoff profile sought in the above question). We use Theorem 1 in a systematic way to derive sharing rules for a Bertrand-type duopoly game and a game considered in Simon and Zame (1990). This is done by linear interpolation across discontinuities, as sketched above. Moreover, we show that Theorem 1 can also be used to prove the main existence result of Simon and Zame (1990) in two different ways. Recently, Jackson et al. (2002) have used endogenous sharing rules to obtain new existence results for games with incomplete information. The model which they consider is considerably more complicated than the one studied here, but in Balder (2004) methods similar to the ones presented here have been used to obtain a partial generalization of the main result in Jackson et al. (2002). The main existence result of Balder (2004) also generalizes the main existence result from Simon and Zame (1990). An earlier account of the results presented here, but in a somewhat less general form, can be found in a 2001 preprint of the present author (Balder 2001).

\section{Mathematical preliminaries}

This section recalls (a) some measure theory, principally weak convergence theory for measures, for which we use Aliprantis and Border (2006) and Neveu (1965) as our main references (see also Billingsley 1968) and (b) some basic notions from Myerson (1991) about games in strategic form. Given a separable metric space $X$, let $\mathcal{B}(X)$ stand for the Borel $\sigma$-algebra on $X$. Let $\operatorname{Prob}(X)$ be the set of all probability measures on $(X, \mathcal{B}(X))$. The weak topology on $\operatorname{Prob}(X)$ is the weakest topology for which the mapping $v \mapsto \int_{X} c \mathrm{~d} v$ is continuous for every bounded and continuous function $c: X \rightarrow \mathbb{R}$; cf. p. 507 of Aliprantis and Border (2006). Since $X$ itself is separable and metric, the $\operatorname{set} \operatorname{Prob}(X)$ is separable and metrizable for the weak topology by Theorem 5.12 of Aliprantis and Border (2006); for instance, the Prohorov metric (see Billingsley 1968) can serve as a metric on $\operatorname{Prob}(X)$. Observe that every subset $X^{\prime}$ of $X$ inherits the metric from $X$ (and the associated relative topology). Moreover, if $X^{\prime} \in \mathcal{B}(X)$, then its Borel $\sigma$-algebra $\mathcal{B}\left(X^{\prime}\right)$ consists precisely of all sets $B \subset X^{\prime}$ with $B \in \mathcal{B}(X)$. Moreover, $\operatorname{Prob}\left(X^{\prime}\right)$ can be identified with the set of all $v \in \operatorname{Prob}(X)$ such that $v\left(X^{\prime}\right)=1$, because any $v \in \operatorname{Prob}\left(X^{\prime}\right)$ has a canonical extension $B \mapsto v\left(B \cap X^{\prime}\right)$ which belongs to $\operatorname{Prob}(X)$. The resulting inclusion $\operatorname{Prob}\left(X^{\prime}\right) \subset \operatorname{Prob}(X)$ means that $\operatorname{Prob}\left(X^{\prime}\right)$ can be equipped with the relative weak topology (see Lemma 15.4 of Aliprantis and Border 2006) and this will always be done tacitly from now on. Because of the metrizability of $\operatorname{Prob}(X)$, the weak topology can also be described in terms of sequential convergence. Note that a sequence $\left\{v_{n}\right\}_{n}:=\left\{v_{n}\right\}_{n \in \mathbb{N}}$ in $\operatorname{Prob}(X)$ converges weakly to $v_{0}$ if and only if the integrals 
$\int_{X} c \mathrm{~d} v_{n}$ converge to $\int_{X} c \mathrm{~d} v_{0}$ for every bounded and continuous function $c: X \rightarrow \mathbb{R}$. Theorem 5.13 in Aliprantis and Border (2006), which figures as the portmanteau theorem in Billingsley (1968), provides several alternative but equivalent definitions of weak (sequential) convergence. As another alternative, as such not included in the cited references, we state the following result for sequences, which could easily be reformulated for generalized sequences so as to be consistent with Theorem 5.13 of Aliprantis and Border (2006).

Proposition 1 Let $\left\{v_{n}\right\}_{n}$ be a sequence in $\operatorname{Prob}(X)$ and let $v_{0} \in \operatorname{Prob}(X)$. Then $\left\{v_{n}\right\}_{n}$ converges weakly to $v_{0}$ if and only if $\lim \inf _{n} \int_{B} q \mathrm{~d} v_{n} \geq \int_{B} q \mathrm{~d} v_{0}$ for every lower semicontinuous $q: X \rightarrow \mathbb{R}$, bounded from below, and for every set $B \in \mathcal{B}(X)$ whose boundary $\partial B:=$ cl $B \backslash$ int $B$ is $v_{0}$-null.

Proof Necessity of weak convergence obviously follows from taking $B=X$ and then applying the limes inferior inequality twice, namely to $q:=c$ and $q:=-c$. Here, $c: X \rightarrow \mathbb{R}$ is an arbitrary bounded and continuous function. Next, we prove sufficiency in two steps.

Step 1: for nonnegative $q$. It is easy to see that $q^{\prime}(x):=q(x) 1_{\text {int } B}(x)$ defines a nonnegative lower semicontinuous function $q^{\prime}: X \rightarrow \mathbb{R}$. So by Theorem 15.5 in Aliprantis and Border (2006) it follows that $\liminf _{n} \int_{X} q^{\prime} \mathrm{d} v_{n} \geq \int_{X} q^{\prime} \mathrm{d} v_{0}$ (note that the additional boundedness from above required in Theorem 15.5 in Aliprantis and Border (2006) can be removed by additional truncation and monotone convergence). Using $1_{B} \geq 1_{\text {int } B}$ on the left and $v_{0}(B \backslash \operatorname{int} B)=0$ on the right, this gives the desired inequality.

Step 2: for general $q$. By boundedness below, there exists $\gamma \in \mathbb{R}$ such that $q^{\prime \prime}:=$ $q+\gamma$ is nonnegative. Step 1 applies to $q^{\prime \prime}$, giving $\lim \inf _{n}\left[\int_{B} q \mathrm{~d} v_{n}+\gamma v_{n}(B)\right] \geq$ $\int_{B} q \mathrm{~d} \nu_{0}+\gamma \nu_{0}(B)$. By part 7 of Theorem 15.3 in Aliprantis and Border (2006) we also have $v_{n}(B) \rightarrow v_{0}(B)$. So the desired inequality follows.

Next, we discuss (relative) compactness for the weak topology. Recall that a subset $M$ of $\operatorname{Prob}(X)$ is defined to be tight if for every $\epsilon>0$ there exists a compact subset $K_{\epsilon}$ of $X$ such that $v\left(X \backslash K_{\epsilon}\right)<\epsilon$ for every $v \in M$. Lemma 15.21 in Aliprantis and Border (2006) guarantees that every tight subset of $\operatorname{Prob}(X)$ is relatively compact (whence also relatively sequentially compact) for the weak topology. Moreover, if the space $X$ is a Polish space (i.e., separable, metric and complete), then the converse is also true by Theorem 15.22 in Aliprantis and Border (2006); in Billingsley (1968), the former result is called Prohorov's theorem and the latter one is called the converse Prohorov theorem.

Further, let $Y$ be another separable metric space; then for any set $D \subset X \times Y$ and any $x \in X$ the section of $D$ at $x$ is defined by $D_{x}:=\{y \in Y:(x, y) \in D\}$. Recall from section III.2 in Neveu (1965) that a transition probability from $X$ into $Y$ is a function $\delta: X \mapsto \operatorname{Prob}(Y)$ such that $x \mapsto \delta(x)(B)$ is $\mathcal{B}(X)$-measurable for every $B \in \mathcal{B}(Y)$. Because $Y$ is a separable metric space, it is easy to show that this measurability property is equivalent to the measurability of the function $\delta: X \mapsto \operatorname{Prob}(Y)$ with respect to the $\sigma$-algebras $\mathcal{B}(X)$ on $X$ and $\mathcal{B}(\operatorname{Prob}(Y))$ on $\operatorname{Prob}(Y)$. To every function $f: X \rightarrow Y$, measurable with respect to $\mathcal{B}(X)$ and $\mathcal{B}(Y)$, there corresponds a unique 
Dirac transition probability $\epsilon_{f}: X \mapsto \operatorname{Prob}(Y)$ which is defined by setting $\epsilon_{f}(x):=$ point probability measure at $f(x)$ for every $x \in X$. According to the Tonelli-Fubini theorem for product measures induced by a measure and a transition probability (see Proposition III.2.1 in Neveu 1965), for a given $\mu \in \operatorname{Prob}(X)$ and a transition probability $\delta: X \rightarrow \operatorname{Prob}(Y)$ a product probability measure $\pi$ on $(X \times Y, \mathcal{B}(X) \otimes \mathcal{B}(Y))$ is determined by $\pi(A \times B):=\int_{A} \delta(x)(B) \mu(\mathrm{d} x)$ for every $A \in \mathcal{B}(X), B \in \mathcal{B}(Y)$ and by that same result

$$
\int_{X \times Y} u \mathrm{~d} \pi=\int_{X}\left[\int_{Y} u(x, y) \delta(x)(\mathrm{d} y)\right] \mu(\mathrm{d} x)
$$

holds for every nonnegative and $\mathcal{B}(X) \otimes \mathcal{B}(Y)$-measurable $u: X \times Y \rightarrow \mathbb{R}$. In particular, for $u:=1_{E}$ with $E \in \mathcal{B}(X \times Y)=\mathcal{B}(X) \otimes \mathcal{B}(Y)$ this gives $\pi(E)=\int_{X} \delta(x)\left(E_{x}\right) \mu(\mathrm{d} x)$. In the special situation with $\delta:=\epsilon_{f}$ for a measurable function $f: X \rightarrow Y$ the above product measure becomes $\pi(A \times B)=\mu\left(A \cap f^{-1}(B)\right)$; then the $Y$-marginal of $\pi$ coincides with the usual image of $\mu$ under $f$, because $\pi(X \times B)=\mu\left(f^{-1}(B)\right)$ holds for every $B \in \mathcal{B}(Y)$. In the converse direction, a classical disintegration result (e.g., see Valadier 1973) states that if $\pi \in \operatorname{Prob}(X \times Y)$ is such that its $Y$-marginal $B \mapsto \pi(X \times B)$, which belongs to $\operatorname{Prob}(Y)$, is tight (as defined above), then there exists a transition probability $\delta: X \rightarrow \operatorname{Prob}(Y)$ such that $\pi(A \times B):=\int_{A} \delta(x)(B) \mu(\mathrm{d} x)$ for every $A \in \mathcal{B}(X), B \in \mathcal{B}(Y)$, where $\mu \in \operatorname{Prob}(X)$ is the $X$-marginal probability $A \mapsto \pi(A \times Y)$. In statistics similar results are known as regular conditional distribution results.

Next, we discuss the supports of weakly convergent sequences of probability measures and their limits. Recall that the support of any probability measure $v$ in $\operatorname{Prob}(X)$ is defined by supp $v:=\cap\{F: F \subset X, F$ closed and $v(F)=1\}$; because we work with a separable metric space $X$, this definition conforms to Aliprantis and Border (2006) by their Theorem 12.14. Recall also that the (sequential) limes superior (in the sense of Kuratowski) of any sequence $\left\{A_{n}\right\}_{n}$ of subsets of $X$ is defined as the set of all limit points of $\left\{A_{n}\right\}_{n}$, i.e., the set of all $x \in X$ for which there exists a subsequence $\left\{A_{n_{k}}\right\}_{k}$ and corresponding points $x_{k}$ in $A_{n_{k}}$ such that $x_{k} \rightarrow x$ for $k \rightarrow \infty$. This set will be denoted by $\operatorname{Ls}_{n} A_{n}$. Since $X$ is a metric space, one has the following representation for this limes superior:

$$
\mathrm{Ls}_{n} A_{n}=\bigcap_{m=1}^{\infty} \mathrm{cl} \bigcup_{n \geq m} A_{n}
$$

Proposition 2 Let $\left\{v_{n}\right\}_{n}$ in $\operatorname{Prob}(X)$ converge weakly to $v_{0}$. Then

$$
\text { supp } v_{0} \subset \operatorname{Ls}_{n} \operatorname{supp} v_{n}
$$

Proof Fix any $m \in \mathbb{N}$ and let $F:=\mathrm{cl} \cup_{n \geq m}$ supp $v_{n}$. Then $X \backslash F$ is open, so Theorem 15.3, part 6, in Aliprantis and Border (2006) implies $v_{0}(X \backslash F) \leq$ $\lim \inf _{n} v_{n}(X \backslash F)=0$, where we also use that $v_{n}(X \backslash F)=0$ for all $n \geq m$. Hence, 
supp $v_{0} \subset F$. In view of the above representation of the limes superior, this finishes the proof.

Proposition 3 Let $v \in \operatorname{Prob}(X)$ and let $f: X \rightarrow Y$ be a continuous mapping into some separable metric space $Y$. Define $\pi \in \operatorname{Prob}(X \times Y)$ by $\pi(A \times B):=$ $v\left(A \cap f^{-1}(B)\right)$ for $A \in \mathcal{B}(X), B \in \mathcal{B}(Y)$. Then supp $\pi=\{(x, f(x)): x \in \operatorname{supp} v\} \subset$ $\{(x, f(x)): x \in X\}=:$ gph $f$.

Proof The set $F:=\{(x, f(x)): x \in \operatorname{supp} v\}$ is closed in $X \times Y$ by the continuity of $f$. Now observe that $\pi$ is the product measure induced by $v$ and the Dirac transition probability $\epsilon_{f}$. By the above Tonelli-Fubini theorem this gives

$$
\pi(F)=\int_{X} \epsilon_{f}(x)\left(F_{X}\right) v(\mathrm{~d} x)=v(\operatorname{supp} v)=1
$$

because $F_{x}=\{f(x)\}$, so the closed set $F$ contains supp $\pi$. To prove the converse, we note that also the set $F^{\prime}:=\{x \in X:(x, f(x)) \in \operatorname{supp} \pi\}$ is closed by continuity of $f$. Since $\pi(\operatorname{supp} \pi)=1$, it follows again by the Tonelli-Fubini theorem that

$$
1=\pi(\operatorname{supp} \pi)=\int_{X} \epsilon_{f}(x)\left((\operatorname{supp} \pi)_{x}\right) v(\mathrm{~d} x) .
$$

So $f(x) \in(\operatorname{supp} \pi)_{x}$ for $v$-almost every $x$ in $X$, which is to say that $x \in F^{\prime}$ for $\nu$-almost every $x$ in $X$. Hence, it follows first that $v\left(F^{\prime}\right)=1$ and then also that supp $\nu \subset F^{\prime}$. By definition of $F^{\prime}$ it now follows that $F \subset \operatorname{supp} \pi$.

Finally, we recall some basic game-theoretical notions (e.g., see Aliprantis et al. 2006 or Myerson 1991). Let $N$ be a natural number and let $I:=\{1, \ldots, N\}$ be the set of players. Let $X_{1}, \ldots, X_{N}$ be $N$ separable metric spaces. Let $X$ stand for the Cartesian product $\Pi_{i \in I} X_{i}$. Below we shall also use $X_{-i}:=\Pi_{j \in I \backslash i} X_{j}$ and related well-known notations from game theory. Let $u_{1}, \ldots, u_{N}$ be $N$ functions from $X$ into $\mathbb{R}$ which are bounded and measurable. By

$$
\Gamma:=\left(I,\left(X_{i}\right)_{i \in I},\left(u_{i}\right)_{i \in I}\right)
$$

we denote the strategic-form game that is defined by giving each player $i \in I$ the action space $X_{i}$ and the payoff function $u_{i}$. A mixed action profile for the above game $\Gamma$ is a vector $\left(\beta_{i}\right)_{i \in I}$ of probability measures $\beta_{i} \in \operatorname{Prob}\left(X_{i}\right), i \in I$. Such a mixed action profile is defined to be a mixed Nash equilibrium profile for $\Gamma$ if

$$
\int_{X} u_{i} \mathrm{~d} \tilde{\beta}=\sup _{x_{i} \in X_{i}} \int_{X_{-i}} u_{i}\left(x_{i}, x_{-i}\right) \tilde{\beta}_{-i}\left(\mathrm{~d} x_{-i}\right) \quad \text { for every } i \in I
$$

and for $\epsilon \geq 0$ it is called a mixed $\epsilon$-Nash equilibrium profile if for every $i \in I$ the above left side, when $\epsilon$ is added to it, is larger than the right side. Here $\tilde{\beta}$ and $\tilde{\beta}_{-i}$ 
stand for the product measures $\times_{i \in I} \beta_{i}:=\beta_{1} \times \cdots \times \beta_{N}$ and $\times_{j \in I \backslash i} \beta_{j}$. On the other hand, a pure Nash equilibrium profile for the above game $\Gamma$ is a vector $\left(y_{i}\right)_{i \in I}$ in $X$ such that

$$
u_{i}(y)=\sup _{x_{i} \in X_{i}} u_{i}\left(x_{i}, y_{-i}\right) \quad \text { for every } i \in I
$$

this is equivalent to $\left(\beta_{i}\right)_{i \in I}$ being a mixed Nash equilibrium profile for $\Gamma$, with $\beta_{i}:=$ point probability measure at $y_{i}$.

\section{Main result}

As in the previous section, let $I:=\{1, \ldots, N\}$ be a set of $N$ players. Let $S_{1}, \ldots, S_{N}$ be $N$ separable, metric and complete spaces. Below we shall write $S:=\Pi_{i \in I} S_{i}$ and the product metric on $S$ is for denoted by $d_{S}$. Let $n \in \mathbb{N}$; the $n$-th stage game is defined by

$$
\Gamma^{(n)}:=\left(I,\left(S_{i}^{(n)}\right)_{i \in I},\left(q_{i}^{(n)}\right)_{i \in I}\right)
$$

Its so-called $n$-th stage action spaces are $S_{1}^{(n)}, \ldots, S_{N}^{(n)}$ and its $n$-th stage payoffs are the functions $q_{1}^{(n)}, \ldots, q_{N}^{(n)}: S^{(n)} \rightarrow \mathbb{R}$, where $S^{(n)}:=\Pi_{i \in I} S_{i}^{(n)}$. We suppose that

$$
S_{i}^{(n)} \in \mathcal{B}\left(S_{i}\right) \text { for every } i \in I \text {, }
$$

i.e., $S_{i}^{(n)}$ is a Borel measurable subset of $S_{i}$ for every $i \in I$. Moreover, for each player $i$ 's sequence $\left\{S_{i}^{(n)}\right\}_{n}$ of staged action spaces in $S_{i}$ we assume

$$
S_{i}=\operatorname{Ls}_{n} S_{i}^{(n)} \quad \text { for every } i \in I
$$

Recall from Sect. 2 that this means that for every $i \in I$ and every $s_{i} \in S_{i}$ there must exist a subsequence $\left\{S_{i}^{\left(n_{k}\right)}\right\}_{k}$ of staged action spaces and corresponding actions $s_{i}^{k} \in S_{i}^{\left(n_{k}\right)}$, such that $s_{i}^{k} \rightarrow s_{i}$ for $k \rightarrow \infty$. Furthermore, we suppose that

$$
q_{i}^{(n)}: S^{(n)} \rightarrow \mathbb{R} \text { is continuous for every } i \in I .
$$

Let $q^{(n)}$ stand for the payoff profile (i.e., vector function) $\left(q_{i}^{(n)}\right)_{i \in I}: S^{(n)} \rightarrow \mathbb{R}^{N}$. For each player $i$ 's sequence $\left\{q_{i}^{(n)}\right\}_{n}$ of staged payoffs we suppose that they are uniformly bounded in the following sense:

$$
C_{i}:=\sup _{n \in \mathbb{N}} \sup _{s \in S^{(n)}}\left|q_{i}^{(n)}(s)\right|<+\infty \quad \text { for every } i \in I .
$$


Theorem 1 For every $n \in \mathbb{N}$ let $\left(\alpha_{1}^{(n)}, \ldots, \alpha_{N}^{(n)}\right)$ be a mixed Nash equilibrium for the game $\Gamma^{(n)}$. Suppose that for every $i \in I$ the sequence $\left\{\alpha_{i}^{(n)}\right\}_{n}$ converges weakly to some probability measure $\beta_{i} \in \operatorname{Prob}\left(S_{i}\right)$. Then under (3)-(5) there exist $N$ measurable functions $q_{1}^{*}, \ldots, q_{N}^{*}: S \rightarrow \mathbb{R}$, with $\sup _{s \in S}\left|q_{i}^{*}(s)\right| \leq C_{i}$ for every $i \in I$, such that $\left(\beta_{i}\right)_{i \in I}$ is a mixed Nash equilibrium profile for the game

$$
\Gamma^{*}:=\left(I,\left(S_{i}\right)_{i \in I},\left(q_{i}^{*}\right)_{i \in I}\right)
$$

and the so-called resolvent payoff profile $q^{*}:=\left(q_{i}^{*}\right)_{i \in I}: S \rightarrow \mathbb{R}^{N}$ has the following properties:

$$
\begin{gathered}
q^{*}(s) \in \operatorname{co}\left(\left(L s_{n} g p h q^{(n)}\right)_{s}\right) \quad \text { for every } s \in S, \\
q^{*}(s) \in \operatorname{co}\left(\left(\operatorname{Ls}_{n}\left\{\left(s^{\prime}, q^{(n)}\left(s^{\prime}\right)\right): s^{\prime} \in \operatorname{supp} \tilde{\alpha}^{(n)}\right\}\right)_{s}\right) \quad \text { for } \tilde{\beta} \text {-a.e. } s \in S,
\end{gathered}
$$

and

$$
\lim _{n \rightarrow \infty} \int_{S^{(n)}} q_{i}^{(n)} \mathrm{d} \tilde{\alpha}^{(n)}=\int_{S} q_{i}^{*} \mathrm{~d} \tilde{\beta} \quad \text { for every } i \in I .
$$

Here, $\tilde{\alpha}^{(n)}:=\alpha_{1}^{(n)} \times \cdots \times \alpha_{N}^{(n)} \in \operatorname{Prob}\left(S^{(n)}\right)$ and $\tilde{\beta}:=\beta_{1} \times \cdots \times \beta_{N} \in \operatorname{Prob}(S)$.

Observe that Theorem 1, which will be proved in Sect. 5, does not contain any explicit compactness condition. Also, we note that the continuity condition (4) is needed for (7), but not for (6) or (8). The theorem is an equilibrium closure result in that it shows the weak limit $\left(\beta_{i}\right)_{i \in I}$ of the sequence $\left\{\left(\alpha_{i}^{(n)}\right)_{i \in I}\right\}_{n}$ to be a Nash equilibrium profile with respect to some payoff profile $q^{*}:=\left(q_{i}^{*}\right)_{i \in I}$ which is tied to the staged payoff profiles in $\left\{q^{(n)}\right\}_{n}$ via (6)-(8). As such, it shows quite some relationship with lower closure results in optimal control and economics (see Balder 1984), of which Fatou's lemma in several dimensions is a well-known specimen. We observe that it is easy to extend the scope of Theorem 1 a little further, namely by merely requiring for a sequence $\epsilon_{n} \rightarrow 0$ in $\mathbb{R}_{+}$that each $\left(\alpha_{1}^{(n)}, \ldots, \alpha_{N}^{(n)}\right)$ be an $\epsilon_{n}$-almost Nash equilibrium for $\Gamma^{(n)}$.

When the counterpart of (6) is sought in Simon and Zame (1990), it cannot be found in explicit form. However, it is evident that the results stated there would lead to

$$
q^{*}(s) \in \operatorname{co}\left(\left(\operatorname{cl} \bigcup_{n=1}^{\infty} \operatorname{gph} q^{(n)}\right)\right) \quad \text { for every } s \in S,
$$

because the right hand side defines the smallest compact-convex-valued upper semicontinuous multifunction that has all $q^{(n)}$ as its measurable selectors. It is important 
to note that this multifunction has larger values than the one defined by the right hand side of (6).

A special case of Theorem 1 occurs when one deals with pure Nash equilibria.

Corollary 1 For every $n \in \mathbb{N}$ let $s^{(n)} \in S^{(n)}$ be a pure Nash equilibrium for the game $\Gamma^{(n)}$. Suppose that the sequence $\left\{s^{(n)}\right\}_{n}$ converges to some vector $t \in S$. Then under (3)-(5) there exist $N$ measurable functions $q_{1}^{*}, \ldots, q_{N}^{*}: S \rightarrow \mathbb{R}$, with $\sup _{s \in S}\left|q_{i}^{*}(s)\right| \leq C_{i}$ for every $i \in I$, such that $t$ is a pure Nash equilibrium profile for the game

$$
\Gamma^{*}:=\left(I,\left(S_{i}\right)_{i \in I},\left(q_{i}^{*}\right)_{i \in I}\right)
$$

and the so-called resolvent payoff profile $q^{*}:=\left(q_{i}^{*}\right)_{i \in I}: S \rightarrow \mathbb{R}^{N}$ has the following properties:

$$
\begin{aligned}
& q^{*}(s) \in \operatorname{co}\left(\left(\operatorname{Ls}_{n} g p h q^{(n)}\right)_{s}\right) \quad \text { for every } s \in S, \\
& q^{*}(t)=\lim _{n} q^{(n)}\left(s^{(n)}\right) .
\end{aligned}
$$

Applications of this result will be given in the following section.

\section{Applications}

As in the previous section, let $I:=\{1, \ldots, N\}$ be a set of $N$ players. In this section we require $S_{1}, \ldots, S_{N}$ to be compact metric spaces, except for Example 3 at the end. As before, we write $S:=\prod_{i \in I} S_{i}$, etc.

\subsection{The Simon-Zame existence result for endogenous sharing rules}

In this section, we show that Theorem 1 implies the main existence result for endogenously shared equilibria in the seminal paper of Simon and Zame (1990) in two different ways: in the first one the staged action spaces are discrete and in the second one they are continuous. In the results in Simon and Zame (1990) properties (6) and (7) are absent. See our comments in connection with (9) above. As we shall observe following our two proofs, this means that the second proof below cannot be given by the methods in Simon and Zame (1990) alone. Following Simon and Zame (1990), let $Q: S \rightarrow 2^{\mathbb{R}^{N}}$ be a multifunction with the following property:

$Q$ is upper semicontinuous on $S$ with nonempty, convex and compact values.

Because $S$ is compact (see the beginning of this section) the above property guarantees that

$$
\text { gph } Q:=\{(s, z): s \in S, z \in Q(s)\} \text { is a compact subset of } S \times \mathbb{R}^{N}
$$


by a result of Berge; see Lemma 17.3 in Aliprantis and Border (2006) and note that upper semicontinuity is called upper hemicontinuity both there and in Simon and Zame (1990). We now use Theorem 1 to derive the following existence result of Simon and Zame (1990) in two different ways.

Theorem 2 There exist $N$ measurable and bounded functions $q_{1}^{*}, \ldots, q_{N}^{*}: S \rightarrow \mathbb{R}$ and a mixed action profile $\left(\beta_{i}\right)_{i \in I} \in \Pi_{i \in I} \operatorname{Prob}\left(S_{i}\right)$ which is a Nash equilibrium for the game

$$
\Gamma^{*}:=\left(I,\left(S_{i}\right)_{i \in I},\left(q_{i}^{*}\right)_{i \in I}\right)
$$

Here the measurable function $q^{*}:=\left(q_{i}^{*}\right)_{i \in I}: S \rightarrow \mathbb{R}^{N}$ is such that $q^{*}(s) \in Q(s)$ for every $s \in S$.

First proof: discretization of $S$. Because the product space $S$ is compact and metric, it is separable. Let $\left\{s^{k}\right\}_{k \in \mathbb{N}}$ be a countable dense subset of $S$ (without loss of generality we may consider only the case where the set $S$ is not finite) and define $S^{(n)}:=\left\{s^{k}\right\}_{k=1}^{n}$ for each $n \in \mathbb{N}$. Then condition (3) holds. Because of (13), the Kunugui-Novikov theorem (this is Theorem 1 in Brown and Purves 1973) implies that $Q$ has a measurable selection $q: S \rightarrow \mathbb{R}^{N}$. Also, by (13) the function $q$ is bounded. Define $q^{(n)}$ to be the restriction of $q$ to the discrete subset $S^{(n)}, n \in \mathbb{N}$; then, evidently, $q^{(n)}$ is continuous on $S^{(n)}$ and condition (5) holds. For $n \in \mathbb{N}$, let the $n$-th stage game $\Gamma^{(n)}$ be defined as in (2). By Nash's original existence result, each game $\Gamma^{(n)}$ has a mixed equilibrium profile $\alpha^{(n)}:=\left(\alpha_{i}^{(n)}\right)_{i \in I}$, with $\alpha_{i}^{(n)} \in \operatorname{Prob}\left(S_{i}^{(n)}\right) \subset \operatorname{Prob}\left(S_{i}\right)$ (recall what was said about such identification in Sect. 2). By compactness of the spaces $S_{i}$, the spaces $\operatorname{Prob}\left(S_{i}\right)$ are compact for the weak topology (use Theorem 15.11 in Aliprantis and Border 2006) and they are also metrizable (recall this from Sect. 2). Thus, there exists a subsequence $\left\{\alpha^{\left(n_{k}\right)}\right\}_{k \in \mathbb{N}}$ such that for each $i \in I$ the sequence $\left\{\alpha_{i}^{\left(n_{k}\right)}\right\}_{k}$ converges weakly to some probability measure $\beta_{i} \in \operatorname{Prob}\left(S_{i}\right)$. By Theorem 1 it now follows that there exist bounded measurable functions $q_{i}^{*}: S \rightarrow \mathbb{R}$ such that $\left(\beta_{i}\right)_{i \in I}$ is a Nash equilibrium for the game $\left(I,\left(S_{i}\right)_{i \in I},\left(q_{i}^{*}\right)_{i \in I}\right)$, where $q^{*}:=\left(q_{i}^{*}\right)_{i \in I}$ has the pointwise property (6). In view of (13), the graph gph $Q$ contains the limes superior set $\operatorname{Ls}_{n}$ gph $q^{(n)}$. Hence, by (6) and the convexity of the values of $Q$, as guaranteed in (12), it follows that $q^{*}(s)$ belongs to $Q(s)$ for every $s \in S$. So the resolvent payoff profile $q^{*}$ is a measurable selection of $Q$.

Second proof: continuous approximation of $Q$. This time, we work with $S^{(n)}=S$ for all $n$. Assumption (12) allows us to apply an approximate continuous selection theorem that goes back to von Neumann; see Theorem 1 on p. 84 of Aubin and Cellina (1984). Hence, there exists a sequence $\left\{q^{(n)}\right\}_{n}$ of continuous functions $q^{(n)}: S \rightarrow \mathbb{R}^{N}$ such that

$$
\lim _{n \rightarrow \infty} \sup _{s \in S} \operatorname{dist}\left(\left(s, q^{(n)}(s)\right), \operatorname{gph} Q\right)=0
$$

where distance is defined with respect to the product metric on $S \times \mathbb{R}^{N}$. In view of (13), it then follows with ease that condition (5) is fulfilled. Moreover, the limes superior set 
$\mathrm{Ls}_{n} \mathrm{gph} q^{(n)}$ is contained in gph $Q$ : indeed, if $\left(s^{k}, z^{k}\right) \rightarrow(\bar{s}, \bar{z})$ for $k \rightarrow \infty$, with $z^{k}=$ $q^{\left(n_{k}\right)}\left(s^{k}\right)$ for all $k$, then the above approximation property gives $\operatorname{dist}((\bar{s}, \bar{z}), \operatorname{gph} Q)=0$, whence $(\bar{s}, \bar{z}) \in$ gph $Q$ by (13). Let $\Gamma^{(n)}, n \in \mathbb{N}$, be defined as in (2), but now with $S^{(n)}:=S$. By the extension of Nash's mixed equilibrium existence result of Glicksberg (1952), each game $\Gamma^{(n)}$ has a mixed equilibrium profile $\alpha^{(n)}:=\left(\alpha_{i}^{(n)}\right)_{i \in I}$. Just as in the previous proof, it now follows that there exists a subsequence $\left\{\alpha^{\left(n_{k}\right)}\right\}_{k \in \mathbb{N}}$ such that for each $i \in I$ the sequence $\left\{\alpha_{i}^{\left(n_{k}\right)}\right\}_{k}$ converges weakly to some $\beta_{i} \in \operatorname{Prob}\left(S_{i}\right)$. By Theorem 1 there exist bounded measurable functions $q_{i}^{*}: S \rightarrow \mathbb{R}$ such that $\left(\beta_{i}\right)_{i \in I}$ is a Nash equilibrium for the game $\Gamma^{*}:=\left(I,\left(S_{i}\right)_{i \in I},\left(q_{i}^{*}\right)_{i \in I}\right)$, where $q^{*}:=\left(q_{i}^{*}\right)_{i \in I}$ has property (6). So from the above inclusion $\operatorname{Ls}_{n}$ gph $q^{(n)} \subset$ gph $Q$ we obtain that $q^{*}(s) \in Q(s)$ for every $s \in S$.

To see the importance of the approximation properties (6)-(7), we observe the following. The first proof above is closely reflected by the original proof in Simon and Zame (1990). It does not use any limit properties of the sequence $\left\{q^{(n)}\right\}_{n}$, because it has the essential feature gph $q^{(n)} \subset \operatorname{gph} Q$ (in the abstract we called this approximation "from within"). In contrast, in the second proof one only has that gph $q^{(n)}$ approximates gph $Q$ in some sense (i.e., approximation "from without"). Thus, a limit property such as (6) is essential for such a proof.

Remark 1 If one starts with an arbitrary nonempty-valued multifunction $\tilde{Q}: S \rightarrow$ $2^{\mathbb{R}^{N}}$ which only satisfies the following requirement:

$$
\bigcup_{s \in S} \tilde{Q}(s) \subset \mathbb{R}^{N} \text { is a bounded set, }
$$

then by setting $Q(s):=\operatorname{co}(\operatorname{clgph} \tilde{Q})_{s}$ one obtains a multifunction $Q$ which meets condition (12). This requires an application of Corollary 11.63 in Moore (1999). In particular, for any bounded function $p: S \rightarrow \mathbb{R}^{N}$ this prescription gives (see Simon and Zame 1990)

$$
Q(s):=Q_{p}(s):=\operatorname{co}(\operatorname{clgph} p)_{s}, \quad s \in S,
$$

by setting $\tilde{Q}(s):=\{p(s)\}$. Of course, then $Q(s)=Q_{p}(s)=\{p(s)\}$ at any continuity point $s$ of $p$.

\subsection{Computation of endogenous sharing equilibria}

Theorem 1 can also be used in actual computations of equilibria with endogenous sharing rules. Here, we shall use linear interpolation to construct the $n$-th stage games, which are still of a continuous nature in order to allow for purification. We give two examples.

Example 1 The present example concerns the computation of the equilibrium in a Bertrand duopoly game $\Gamma$. Such equilibria can, of course, be determined in an ad hoc fashion, but the present approach offers the attraction of being quite systematic. 
To make our calculations easy, we shall assume linear demand and per-unit production $\operatorname{cost} c$. More elaborate versions can be produced fairly easily. Let $a>c$; the profit of firm $i \in I:=\{1,2\}$ is supposed to be $p_{i}\left(s_{1}, s_{2}\right):=\left(s_{i}-c\right)\left(a-s_{i}\right)$ if $s_{i}<s_{j}$ and $p_{i}\left(s_{1}, s_{2}\right):=0$ if $s_{i}>s_{j}$. Here, $j=2$ if $i=1$ and $j=1$ if $i=2$ and $s_{i}, s_{j}$ are the price variables; i.e., $s_{j}:=s_{-i}$. In addition, for obvious reasons the price variables must satisfy $s_{i}, s_{j} \geq 0$ and $s_{i}, s_{j} \leq a$. A discontinuity occurs when $s_{i}=s_{j}$, i.e., when both firms charge the same price. It is standard in the literature to allocate total demand evenly in this case, causing profits to be $p_{i}\left(s_{1}, s_{1}\right):=\frac{1}{2}\left(s_{1}-c\right)\left(a-s_{1}\right)$, but we note that there is no compelling economic reason for such a division of the demand. In any case, this is irrelevant for the payoff correspondence, since (14) gives here

$$
Q_{p}\left(s_{1}, s_{1}\right):=\left\{\left(\lambda\left(s_{1}-c\right)\left(a-s_{1}\right),(1-\lambda)\left(s_{1}-c\right)\left(a-s_{1}\right)\right): 0 \leq \lambda \leq 1\right\} .
$$

Here $\lambda$, the fraction of the total demand that goes to firm 1 , forms an additional parameter of the model. As follows by what was observed in Remark 1 about the continuity points of $p$, we have $Q_{p}(s)=\{p(s)\}$ in all other points $s$ of $S:=[0, a]^{2}$. We introduce an approximation by setting $S^{(n)}:=S$ and defining the continuous function $q^{(n)}: S \rightarrow \mathbb{R}^{2}$ by linear interpolation across the discontinuity on the diagonal, i.e.,

$$
q_{i}^{(n)}\left(s_{i}, s_{j}\right):= \begin{cases}p_{i}\left(s_{i}, s_{j}\right) & \text { if } s_{i} \leq s_{j}-\frac{1}{n} \text { or } s_{i} \geq s_{j}+\frac{1}{n} \\ v s_{i}+w & \text { if } s_{j}-\frac{1}{n}<s_{i}<s_{j}+\frac{1}{n}\end{cases}
$$

Here $v$ and $w$ are determined by $v\left(s_{j}-\frac{1}{n}\right)+w=p_{i}\left(s_{j}-\frac{1}{n}, s_{j}\right)$ and $v\left(s_{j}+\frac{1}{n}\right)+w=$ $p_{i}\left(s_{j}+\frac{1}{n}, s_{j}\right)=0$ (i.e., $v=-\frac{n}{2}\left(s_{j}-\frac{1}{n}-c\right)\left(a-s_{j}+\frac{1}{n}\right)$ and $w=\frac{n}{2}\left(s_{j}-\frac{1}{n}-c\right)(a-$ $\left.\left.s_{j}+\frac{1}{n}\right)\left(s_{j}+\frac{1}{n}\right)\right)$. It is easy to check that this interpolation scheme has the following property: if $s_{j}-\frac{1}{n}<s_{i}<s_{j}+\frac{1}{n}$ then the distance of $\left(s_{i}, v s_{i}+w\right)$ to gph $Q_{p}$ is at most $\frac{2}{n}$. This determines the $n$-th stage game $\Gamma^{(n)}$. To find its Nash equilibria, we may use purification since each $q_{i}^{(n)}\left(s_{i}, s_{j}\right)$ is quasi-concave in the variable $s_{i}$. In pure actions the best reply correspondence $B_{i}^{(n)}:[0, a] \rightarrow 2^{[0, a]}$ for player $i$ in the $n$-th approximating game is given by

$$
B_{i}\left(s_{j}\right):= \begin{cases}\left\{s_{i} \in[0, a]: s_{i} \geq s_{j}+\frac{1}{n}\right\} & \text { if } s_{j} \leq c+\frac{1}{n} \\ \left\{s_{j}-\frac{1}{n}\right\} & \text { if } c+\frac{1}{n}<s_{j} \leq \frac{a+c}{2}+\frac{1}{n} \\ \left\{\frac{a+c}{2}\right\} & \text { if } s_{j}>\frac{a+c}{2}+\frac{1}{n}\end{cases}
$$

The fixed points of this correspondence are easily seen to form the following set $S_{0}$ : the union of all pairs $\left(s_{2}+\frac{1}{n}, s_{2}\right)$ with $s_{2} \in\left(c, c+\frac{1}{n}\right]$ and all pairs $\left(s_{1}, s_{1}+\frac{1}{n}\right)$ with $s_{1} \in\left(c, c+\frac{1}{n}\right]$. Evidently, no matter which equilibrium pairs $s^{(n)}:=\left(s_{1}^{(n)}, s_{2}^{(n)}\right)$ of pure actions we choose at stage $n$, they always converge to $(c, c)$ for $n \rightarrow \infty$. By Corollary $1(c, c)$ is a pure Nash equilibrium for a game $\Gamma^{*}$ with a resolvent payoff profile $q^{*}:[0, a]^{2} \rightarrow \mathbb{R}^{2}$ for which $q^{*}\left(s_{1}, s_{2}\right)=p\left(s_{1}, s_{2}\right)$ if $s_{1} \neq s_{2}$ (namely, by applying (10)) and $q^{*}(c, c)=(0,0)$ (by (11)). Together, these tell us the following: $0=q_{1}^{*}(c, c) \geq q_{1}^{*}\left(s_{1}, c\right)$ for every $s_{1} \in[0, a]$ and $0=q_{2}^{*}(c, c) \geq q_{2}^{*}\left(c, s_{2}\right)$ for every 
$s_{2} \in[0, a]$. This outcome agrees with the classical one, even though the precise form of profit sharing when equal prices are chosen was left unspecified.

Example 2 This example, the California-Oregon psychologists' game, already appeared in Simon and Zame (1990). It is interesting to contrast the systematic application below with the rather heuristic "approximation from within" arguments in both Simon and Zame (1990) and pp. 147-148 of Myerson (1991). Consider the following location game in the spirit of Hotelling (1929). It takes place along a section of interstate highway 5 , which is represented by the interval $[0,4]$. The action space of player 1 , the psychologist from California, is $S_{1}:=[0,3]$ (i.e., the Californian highway stretch), and for player 2, the psychologist from Oregon, the action space is $S_{2}:=[3,4]$, which stands for the Oregon part of the highway. The payoff for player 1 is

$$
p_{1}\left(s_{1}, s_{2}\right):= \begin{cases}\frac{s_{1}+s_{2}}{2} & \text { if } s_{1}<s_{2} \\ 2 & \text { if } s_{1}=s_{2}=3\end{cases}
$$

Player 2 has payoff $p_{2}\left(s_{1}, s_{2}\right):=4-p_{1}\left(s_{1}, s_{2}\right)$. Clearly, these functions are discontinuous at the point $\left(s_{1}, s_{2}\right)=(3,3)$, which corresponds to the California-Oregon border. In this example the formula (14) gives

$$
Q_{p}\left(s_{1}, s_{2}\right):= \begin{cases}\left\{\left(z_{1}, 4-z_{1}\right): z_{1} \in[2,3]\right\} & \text { if } s_{1}=s_{2}=3 \\ \left\{\left(p_{1}\left(s_{1}, s_{2}\right), p_{2}\left(s_{1}, s_{2}\right)\right)\right\} & \text { otherwise }\end{cases}
$$

An $n$-th stage game $\Gamma^{(n)}$ is obtained by setting $S^{(n)}:=S=[0,3] \times[3,4]$ for all $n$ and by defining

$$
q_{1}^{(n)}\left(s_{1}, s_{2}\right):= \begin{cases}\left(\frac{1}{2}-n\right) s_{1}+\frac{1}{2}+3 n & \text { if } 3-\frac{1}{n} \leq s_{1} \leq 3 \text { and } s_{2}=3 \\ \frac{s_{1}+s_{2}}{2} & \text { otherwise }\end{cases}
$$

for player 1 and $q_{2}^{(n)}\left(s_{1}, s_{2}\right):=4-q_{1}^{(n)}\left(s_{1}, s_{2}\right)$ for player 2 . This describes a standard linear interpolation of $p$ by $q^{(n)}:=\left(q_{1}^{(n)}, q_{2}^{(n)}\right)$ and it is easy to see that $\sup _{s \in S} \operatorname{dist}\left(\left(s, q^{(n)}(s)\right)\right.$, gph $\left.Q_{p}\right) \leq \frac{1}{n} \rightarrow 0$. Each approximating game has continuous and quasi-concave payoff functions, so it has a Nash equilibrium in pure actions. In fact, standard computation of the best reply correspondence for player 1 gives $B_{1}^{(n)}\left(s_{2}\right)=\{3\}$ if $s_{2}>3$ and $B_{1}^{(n)}\left(s_{2}\right)=\left\{3-\frac{1}{n}\right\}$ if $s_{2}=3$. For player 2 the best reply correspondence is given by $B_{2}^{(n)}\left(s_{1}\right)=\{3\}$. So the unique pure Nash equilibrium for the $n$-th approximating game is $s^{(n)}:=\left(s_{1}^{(n)}, s_{2}^{(n)}\right)=\left(3-\frac{1}{n}, 3\right)$, which is also immediately obvious. Since $\left(s_{1}^{(n)}, s_{2}^{(n)}\right) \rightarrow(3,3)$ and $\left(q_{1}^{(n)}\left(s^{(n)}\right), q_{2}^{(n)}\left(s^{(n)}\right)\right)=\left(3-\frac{1}{2 n}, 1+\frac{1}{2 n}\right) \rightarrow$ $(3,1)$ for $n \rightarrow \infty$, it follows from Corollary 1 that $(3,3)$ is a pure Nash equilibrium profile for a game $\Gamma^{*}$ with payoff profile $q^{*}:[0,3] \times[3,4] \rightarrow \mathbb{R}^{2}$ for which $q^{*}\left(s_{1}, s_{2}\right)=p\left(s_{1}, s_{2}\right)$ if $s_{1} \neq s_{2}$ (namely, by applying $\left.(10)\right)$ and $q^{*}(3,3)=(3,1)$ (by (11)). Thus, in the single discontinuity point $(3,3)$ the resolvent payoff profile $q^{*}$ prescribes the psychologists to divide their patients according to the ratio $3: 1$. Of course, 
this could also have been observed in an ad hoc manner from the fact that $\left(s_{1}^{(n)}, s_{2}^{(n)}\right)$ is a pure Nash equilibrium for the $n$-th approximating game (or even in a blitz manner from the fact that $\left(p_{1}, p_{2}\right)$ has a single removable discontinuity at $\left.(3,3)\right)$, but, again, we point out that the present interpolation method has the appeal of being systematic.

We conclude this section by applying Theorem 1 to a simple situation with noncompact action spaces, i.e., a situation to which the results in Simon and Zame (1990) do not apply for another reason than the ones stated before.

Example 3 Consider a two-player game with action spaces $S_{i}^{(n)}:=S_{i}:=\mathbb{Z}_{+}, i=1,2$. For $n \in \mathbb{N}$ we define as follows. Let $q_{1}^{(n)}\left(s_{1}, s_{2}\right):=1$ if $s_{1} \in\{0, n\}$ and $q_{1}^{(n)}\left(s_{1}, s_{2}\right):=0$ otherwise. Let $q_{2}^{(n)}\left(s_{1}, s_{2}\right):=0$. Let $\alpha_{1}^{(n)} \in \operatorname{Prob}\left(S_{1}\right)$ be the probability measure with support $\{0, n\}$, determined by $\alpha_{1}^{(n)}(\{0\})=1-n^{-1}$ and $\alpha_{1}^{\{n\}}(\{n\})=n^{-1}$. Also, let $\alpha_{2}^{(n)}$ be the probability measure $\epsilon_{0}$ concentrated at 0 . Then for $i=1,2$ the sequence $\left\{\alpha_{i}^{(n)}\right\}_{n}$ converges weakly to the probability measure $\beta_{i}:=\epsilon_{0}$. Trivially, for every $n \in \mathbb{N}$ the pair $\left(\alpha_{1}^{(n)}, \alpha_{2}^{(n)}\right)$ is a mixed Nash equilibrium for the game $\Gamma^{(n)}:=\left(1,2,\left(S_{i}^{(n)}\right)_{i=1}^{2},\left(q_{i}^{(n)}\right)_{i=1}^{2}\right)$. Now

$$
\operatorname{Ls}_{n} \operatorname{gph} q^{(n)}=\left\{\left(0, s_{2}, 1,0\right): s_{2} \in \mathbb{Z}_{+}\right\} \cup\left\{\left(s_{1}, s_{2}, 0,0\right): s_{1}, s_{2} \in \mathbb{Z}_{+}, s_{1} \neq 0\right\}
$$

Hence, (6) amounts to $q^{*}\left(s_{1}, s_{2}\right)=(0,0)$ for $s_{1} \neq 0$ and $q^{*}\left(s_{1}, s_{2}\right)=(1,0)$ for $s_{1}=0$. It is easy to see that $\left(\beta_{1}, \beta_{2}\right)=\left(\epsilon_{0}, \epsilon_{0}\right)$ is indeed a mixed Nash equilibrium profile for $q^{*}$ and that $\int_{\mathbb{Z}_{+}} q^{(n)} \mathrm{d} \tilde{\alpha}^{(n)}=(1,0)=\int_{\mathbb{Z}_{+}} q^{*} \mathrm{~d} \tilde{\beta}$ for all $n \in \mathbb{N}$.

\section{Proof of Theorem 1}

Let $\left\{\left(q^{(n)}, \alpha^{(n)}\right)\right\}_{n}$ be as in the statement of Theorem 1 . We abbreviate by writing $G^{(n)}:=\left\{\left(s, q^{(n)}(s)\right): s \in \operatorname{supp} \tilde{\alpha}^{(n)}\right\}$. Also, let $Z$ stand for the compact set $\Pi_{i \in I}\left[-C_{i}, C_{i}\right] \subset \mathbb{R}^{N}$, where $C_{i}$ is as defined in (5). Let us define $\pi^{(n)}(A \times B):=$ $\tilde{\alpha}^{(n)}\left(A \cap\left(q^{(n)}\right)^{-1}(B)\right)$ for $A \in \mathcal{B}(S)$ and $B \in \mathcal{B}(Z)$; this completely determines the product probability measure $\pi^{(n)} \in \operatorname{Prob}(S \times Z)$, because the measurable rectangles $A \times B$ generate $\mathcal{B}(S \times Z)$. Observe already that supp $\pi^{(n)}=G^{(n)}$ for every $n \in \mathbb{N}$ (apply Proposition 3).

Lemma 1 The sequence $\left\{\pi^{(n)}\right\}_{n}$ contains a subsequence $\left\{\pi^{\left(n^{\prime}\right)}\right\}_{n^{\prime}}$ which converges weakly to some limit probability measure $\pi^{*} \in \operatorname{Prob}(S \times Z)$, which is such that

$$
\operatorname{supp} \pi^{*} \subset L^{\prime}:=\operatorname{Ls}_{n} G^{(n)} \subset \operatorname{Ls}_{n} \mathrm{gph} q^{(n)}=: L .
$$

Proof Let us prove that the sequence $\left\{\pi^{(n)}\right\}_{n}$ is tight (see Sect. 2). First, by Theorem 3.2 of Billingsley (1968) (see Lemma 3.4 of Aliprantis et al. 2006 for a proof in the style of Aliprantis and Border 2006) the sequence $\left\{\tilde{\alpha}^{(n)}\right\}_{n}$ of product probabilities converges weakly to the product probability $\tilde{\beta}$. Hence, the sequence $\left\{\tilde{\alpha}^{(n)}\right\}_{n}$ is tight (apply the converse Prohorov theorem, see Sect. 2). Hence, for every $\epsilon>0$ there exists 
a compact $K_{\epsilon} \subset S$ such that $\sup _{n} \tilde{\alpha}^{(n)}\left(S \backslash K_{\epsilon}\right) \leq \epsilon$. Define $K_{\epsilon}^{\prime}:=K_{\epsilon} \times Z$; this is a compact set and by definition of $\pi^{(n)}$ we have $\pi^{(n)}\left((S \times Z) \backslash K_{\epsilon}^{\prime}\right)=\tilde{\alpha}^{(n)}\left(S \backslash K_{\epsilon}\right) \leq \epsilon$. This proves that $\left\{\pi^{(n)}\right\}_{n}$ is tight. So the convergence result follows by Lemma 15.21 of Aliprantis and Border (2006), i.e., by Prohorov's theorem as recalled in Sect. 2. The final statement follows from Proposition 2.

To save on notation, we shall pretend from now on without loss of generality that the sequence $\left\{\pi^{(n)}\right\}_{n}$ converges as a whole to some $\pi^{*} \in \operatorname{Prob}(S \times Z)$.

Lemma 2 The probability measure $\pi^{*}$ can be decomposed as follows: there exists a transition probability $\delta^{*}: S \rightarrow \operatorname{Prob}(Z)$ such that $\pi^{*}(A \times B)=\int_{A} \delta^{*}(s)(B) \tilde{\beta}(\mathrm{d} s)$ for every $A$ in $\mathcal{B}(S)$ and $B$ in $\mathcal{B}(Z)$ Moreover, $\delta^{*}$ can be chosen in such a way that

$$
\delta^{*}(s)\left(L_{s}\right)=1 \text { for every } s \in S \text { and } \delta^{*}(s)\left(L_{s}^{\prime}\right)=1 \text { for } \tilde{\beta} \text {-a.e. } s \in S \text {. }
$$

Proof Step 1: From Lemma 1 and the definition of weak convergence it follows that $\tilde{\beta}$ is the $S$-marginal of $\pi^{*}$ (consider bounded continuous functions $c(s, z)$ on $S \times Z$ which do not depend on the variable $z$ ). For $\pi^{*}$ we can now invoke the disintegration result as recalled in Sect. 2 to obtain a transition probability $\eta^{*}: S \rightarrow \operatorname{Prob}(Z)$ with $\pi^{*}(A \times B)=\int_{A} \eta^{*}(s)(B) \tilde{\beta}(\mathrm{d} s)$ for all $A$ and $B$. Then the above supp $\pi^{*} \subset L^{\prime}$ gives $\eta^{*}(s)\left(L_{s}^{\prime}\right)=1$ for $\tilde{\beta}$-a.e. $s$ in $S$, by the same Fubini-Tonelli result as used before (i.e., by Proposition III.2.1 in Neveu 1965). Explicitly, this means that there is a $\tilde{\beta}$-null set $M \subset S$ such that $\eta^{*}(s)\left(L_{s}^{\prime}\right)=1$ for every $s \in S \backslash M$.

Step 2: We finish the proof by modifying $\eta^{*}$ on the null set $M$. By formula (1), the set $L$ is closed, so it is certainly a $\mathcal{B}(S) \otimes \mathcal{B}(Z)$-measurable subset of the Polish space $S \times Z$. Also, $L$ has nonempty and compact $s$-sections by (3) and the compactness of $Z$. So by the Kunugui-Novikov theorem in Brown and Purves (1973) there exists a Borel measurable function $\hat{q}: S \rightarrow Z$ with $\hat{q}(s) \in L_{s}$ for every $s \in S$. The desired transition probability $\delta^{*}$ is now obtained by defining

$$
\delta^{*}(s)(B):= \begin{cases}\eta^{*}(s)(B) & \text { if } s \in S \backslash M \\ 1 & \text { if } s \in M \text { and } \hat{q}(s) \in B \\ 0 & \text { if } s \in M \text { and } \hat{q}(s) \notin B\end{cases}
$$

Because $M$ is $\tilde{\beta}$-null, replacing $\eta^{*}$ by $\delta^{*}$ does not affect the identity established in step 1 and $\delta^{*}(s)\left(L_{s}\right)=1$ holds for every $s \in S$ by the construction of $\delta^{*}$.

For $s \in S$ we can interpret $\delta^{*}(s)$ as the conditional distribution of the payoff vector, given that $s$ is the outcome of the players' actions under the joint distribution $\pi^{*}$ on action-payoff pairs. In the next lemma we take the corresponding conditional expectation.

Lemma 3 For every $s \in S$ the vector integral $q^{* *}(s):=\int_{Z} z \delta^{*}(s)(\mathrm{d} z) \in Z$ defines an element in co $L_{s}$. Moreover, the mapping $q^{* *}: S \rightarrow Z$, thus defined, is measurable and it has the following additional property:

$$
q^{* *}(s) \in \operatorname{co} L_{s}^{\prime} \text { for } \tilde{\beta} \text {-a.e. } s \text { in } S .
$$


Proof The set $Z$ is bounded, so for each $s \in S$ the existence of the integral $q_{i}^{* *}(s):=$ $\int_{Z} z_{i} \delta^{*}(s)(\mathrm{d} z)$ for each $i \in I$ is elementary. Moreover, the measurability of each mapping $s \mapsto q_{i}^{* *}(s)$ follows by Proposition III.2.1 in Neveu (1965). By a well-known property of expectations (see Pfanzagl 1974), it follows directly from Lemma 2 that $q^{* *}(s) \in \operatorname{co} L_{s}$ for every $s \in S$ and that (15) holds.

Lemma 4 a. For every $i \in I$ there exists $N_{i}$ in $\mathcal{B}\left(S_{i}\right), \beta_{i}\left(N_{i}\right)=0$, such that for every $s_{i} \in S_{i} \backslash N_{i}$

$$
\int_{S} q_{i}^{* *}(s) \tilde{\beta}(\mathrm{d} s) \geq \int_{S_{-i}} q_{i}^{* *}\left(s_{i}, s_{-i}\right) \tilde{\beta}_{-i}\left(\mathrm{~d} s_{-i}\right) .
$$

b. $\lim _{n \rightarrow \infty} \int_{S^{(n)}} q_{i}^{(n)} \mathrm{d} \tilde{\alpha}^{(n)}=\int_{S} q_{i}^{* *} \mathrm{~d} \tilde{\beta}$ for every $i \in I$.

Proof Fix $i \in I$. Let $B \in \mathcal{B}\left(S_{i}\right)$ be arbitrary. Then, obviously, for every $n \in \mathbb{N}$

$$
\alpha_{i}^{(n)}(B) \int_{S} q_{i}^{(n)} \mathrm{d} \tilde{\alpha}^{(n)} \geq \int_{B \times S_{-i}} q_{i}^{(n)} \mathrm{d} \tilde{\alpha}^{(n)},
$$

because $\alpha^{(n)}$ is a mixed Nash equilibrium profile with $\alpha_{i}^{(n)}\left(S_{i}^{(n)}\right)=1$. We claim that

$$
\beta_{i}(B) \int_{S} q_{i}^{* *} \mathrm{~d} \tilde{\beta} \geq \int_{B \times S_{-i}} q_{i}^{* *} \mathrm{~d} \tilde{\beta} .
$$

First, suppose in addition that $\beta_{i}(\partial B)=0$. Then also $\pi^{*}\left(\partial B \times S_{-i} \times Z\right)=\beta_{i}(\partial B)=0$. The definition of $\pi^{(n)}$ gives

$$
\int_{B \times S_{-i}} q_{i}^{(n)} \mathrm{d} \tilde{\alpha}^{(n)}=\int_{B \times S_{-i} \times Z} z_{i} \pi^{(n)}(\mathrm{d}(s, z))
$$

for every $n \in \mathbb{N}$. So by Proposition 1 , applied to both $(s, z) \mapsto z_{i}$ and $(s, z) \mapsto-z_{i}$, we obtain

$$
\lim _{n} \int_{B \times S_{-i}} q_{i}^{(n)} \mathrm{d} \tilde{\alpha}^{(n)}=\int_{B \times S_{-i} \times Z} z_{i} \pi^{*}(\mathrm{~d}(s, z)) .
$$

Observe that

$$
\int_{B \times S_{-i} \times Z} z_{i} \pi^{*}(\mathrm{~d}(s, z))=\int_{B \times S_{-i}}\left[\int_{Z} z_{i} \delta^{*}(s)(\mathrm{d} z)\right] \tilde{\beta}(\mathrm{d} s)=\int_{B \times S_{-i}} q_{i}^{* *} \mathrm{~d} \tilde{\beta},
$$

by Lemmas 2 and 3. Combined with the previous line this already proves part b by setting $B:=S_{i}$. We also get $\lim _{n} \alpha_{i}^{(n)}(B)=\beta_{i}(B)$ (apply part 7 of Theorem 15.3 in 
Aliprantis and Border (2006) or apply Proposition 1). So (18) follows from (17) for such special $B$.

The next step in proving part a consists of proving the validity of (18) for any closed $B \subset S_{i}$. If $B$ is closed we let $B^{\epsilon}$ be the set of all $s \in S_{i}$ whose distance to $B$ is (strictly) less than $\epsilon>0$. The boundaries of the sets $B^{\epsilon}, \epsilon>0$, which are all disjoint, can have positive $\beta_{i}$-measure for at most countably many $\epsilon$. So for all other $\epsilon$ the set $B^{\epsilon}$ is of the type for which (18) was shown to hold in the previous step. By taking a countable sequence $\left\{\epsilon_{k}\right\}_{k}$ of these, with $\epsilon_{k} \rightarrow 0$, the claimed inequality (18) then follows from the fact that the intersection of all $B^{\epsilon_{k}}, k \in \mathbb{N}$ is the set $B$ (this is true by the closedness of $B$ ). As our final step, we extend the validity of (18) to any $B \in \mathcal{B}\left(S_{i}\right)$. The probability measure $\beta_{i}$ on $S_{i}$ is normal by Theorem 12.5 of Aliprantis and Border (2006) (note that in Theorem 1.1 of Billingsley (1968) this property is called regularity). So, since $B$ is Borel, there exists for every $\epsilon>0$ a closed subset $F^{\epsilon}$ of $B$ with $\beta_{i}\left(B \backslash F^{\epsilon}\right)<\epsilon$. By the above, for each $\epsilon>0$ the inequality (18) holds if we replace $B$ by the closed set $F^{\epsilon}$; hence the validity of (18) for $B$ itself follows by a simple approximation argument. This concludes the proof of the claim above: we know now that (18) holds for any Borel set $B \in \mathcal{B}\left(S_{i}\right)$. From this, (16) follows immediately by standard measure theory.

Lemma 5 For every $i \in I$ the multifunction $s \mapsto \operatorname{argmin}_{z \in L_{s}} z_{i}$ has a measurable selection $\hat{q}^{i}: S \rightarrow Z$.

Proof By formula (1) and compactness of $Z$, the set $L_{S}$ is a nonempty and compact. Hence, the set $\operatorname{argmin}_{z \in L_{s}} z_{i}$ is nonempty for every $s \in S$ (apply the Weierstrass theorem). Fix $i \in I$ and let $\phi_{i}(s):=\inf _{z \in L_{s}} z_{i}$. Using compactness of $Z$, it is standard to prove that $\phi_{i}$ is upper semicontinuous on $S$. Also, by formula (1), the set $L$ is closed in $S \times Z$. Hence, $\left\{(s, z) \in L: z_{i} \leq \phi_{i}(s)\right\}$ is closed and clearly its section at $s$, which is $\operatorname{argmin}_{z \in L_{s}} z_{i}$, is compact for every $s \in S$. The stated result therefore follows from the Kunugui-Novikov measurable selection theorem in Brown and Purves (1973).

Final steps of the proof of Theorem 1: construction of a modification of $q^{* *}$. We shall follow the reasoning in Simon and Zame (1990) to produce a suitable modification of $q^{* *}$ as defined in Lemma 3. Recall the meaning of the $\beta_{i}$-null sets $N_{i}, i \in I$ from Lemma 4. For each $i \in I$, let $\hat{q}^{i}$ be the measurable selection of $s \mapsto \operatorname{argmin}_{z \in L_{s}} z_{i}$ whose existence is guaranteed by Lemma 5. For $i \in I$ let $U^{i} \subset S$ be the Borel set of all $s \in S$ for which $s_{i} \in N_{i}$ but $s_{j} \notin N_{j}$ for all $j \neq i$. Let $U:=\cup_{k \in I} U^{k}$. Then $U$ is evidently $\tilde{\beta}$-null. We define the Borel measurable and bounded function $q^{*}: S \rightarrow Z$, with $q^{*}(s) \in L_{s}$ for every $s \in S$ (use Lemmas 3 and 5), as follows:

$$
q^{*}(s):= \begin{cases}q^{* *}(s) & \text { if } s \notin U, \\ \hat{q}^{i}(s) & \text { if } s \in U^{i} \subset U, i \in I\end{cases}
$$

Note that this function is $\tilde{\beta}$-almost everywhere equal to $q^{* *}$, so by Lemma 3 it has $q^{*}(s) \in\left(\operatorname{Ls}_{n} \text { gph } G^{(n)}\right)_{s}$ for $\tilde{\beta}$-a.e. $s$ in $S$. This proves (7). Fix any $i \in I$. Because every $U^{k}$ is a $\tilde{\beta}$-null set, we have the identity $\int_{S} q_{i}^{*} \mathrm{~d} \tilde{\beta}=\int_{S} q_{i}^{* *} \mathrm{~d} \tilde{\beta}$. Together with 
Lemma 4.b, this proves (8). Moreover, this identity also implies that to prove (6) we must actually prove

$$
\int_{S} q_{i}^{* *} \mathrm{~d} \tilde{\beta} \geq \int_{S_{-i}} q_{i}^{*}\left(s_{i}, \cdot\right) \mathrm{d} \tilde{\beta}_{-i}=\int_{U_{s_{i}}} q_{i}^{*}\left(s_{i}, \cdot\right) \mathrm{d} \tilde{\beta}_{-i}+\int_{S_{-i} \backslash U_{s_{i}}} q_{i}^{* *}\left(s_{i}, \cdot\right) \mathrm{d} \tilde{\beta}_{-i} .
$$

for any fixed $s_{i} \in S_{i}$. Here, $U_{s_{i}}$ denotes the section of $U$ at $s_{i}$, i.e., set of all $s_{-i} \in S_{-i}$ such that $\left(s_{i}, s_{-i}\right) \in U$. We distinguish between two cases:

Case 1: $s_{i} \notin N_{i}$. In this case $\int_{S} q_{i}^{* *} \mathrm{~d} \tilde{\beta} \geq \int_{S_{-i}} q_{i}^{* *}\left(s_{i}, \cdot\right) \mathrm{d} \tilde{\beta}_{-i}$ holds by Lemma 4. Also, in this case $s_{-i} \in U_{s_{i}}$ is equivalent to $\left(s_{i}, s_{-i}\right) \in \cup_{k \neq i} U^{k}$. So $U_{s_{i}}$ is $\tilde{\beta}_{-i}$-null, because $\tilde{\beta}_{-i}$ has each $\beta_{k}, k \neq i$, as one of its factors. Then (19) follows directly from the previous inequality.

Case 2: $s_{i} \in N_{i}$. In this case $U_{s_{i}}$ is precisely the set of all $s_{-i} \in S_{-i}$ such that $\left(s_{i}, s_{-i}\right) \in U^{i}$. We note that $s_{-i} \notin U_{s_{i}}$, combined with the previous line, means that there exists $j \neq i$ such that $s_{j} \in N_{j}$. Because $\tilde{\beta}_{-i}$ has $\beta_{j}$ as one of its factors, this causes $S_{-i} \backslash U_{s_{i}}$ to $\tilde{\beta}_{-i}$-null. In particular, the second integral on the right in (19) is zero. By the definition of the resolvent payoff profile $q^{*}$ and the above identity $U_{s_{i}}=U_{s_{i}}^{i}$, the first integral on the right in (19) equals $\int_{U_{s_{i}}^{i}} \hat{q}^{i}\left(s_{i}, \cdot\right) \mathrm{d} \tilde{\beta}_{-i}$, which is also equal to $\int_{S_{-i}} \hat{q}^{i}\left(s_{i}, \cdot\right) \mathrm{d} \tilde{\beta}_{-i}$ by $\tilde{\beta}_{-i}\left(S_{-i} \backslash U_{s_{i}}^{i}\right)=0$, as just demonstrated. So (19) now follows from $\hat{q}^{i}(s) \in \operatorname{argmin}_{z \in L_{s}} z_{i}$ (by Lemma 5) and $q^{* *}(s) \in L_{S}$ for all $s \in S$ (by Lemma 3), which imply $\hat{q}_{i}^{i} \leq q_{i}^{* *}$.

Open Access This article is distributed under the terms of the Creative Commons Attribution Noncommercial License which permits any noncommercial use, distribution, and reproduction in any medium, provided the original author(s) and source are credited.

\section{References}

Aliprantis, C.D., Border, K.C.: Infinite Dimensional Analysis: a Hitchhiker's Guide (3rd edn.). Berlin: Springer (2006)

Aliprantis, C.D., Glycopantis, D., Puzello, D.: The joint continuity of the expected payoff functions. J Math Econ 42, 121-130 (2006)

Aubin, J.-P., Cellina, A.: Differential Inclusions. Berlin: Springer (1984)

Balder, E.J.: A general approach to lower semicontinuity and lower closure in optimal control theory. SIAM J Control Optim 22, 570-598 (1984)

Balder, E.J.: An equilibrium existence result for games with incomplete information and indeterminate outcomes. J Math Econ 40, 297-320 (2004)

Balder, E.J.: On Equilibria for Discontinuous Games: Nash Approximation Schemes. Preprint no. 1209. Department of Mathematics, University of Utrecht (2001)

Billingsley, P.: Convergence of Probability Measures. New York: Wiley (1968)

Brown, L.D., Purves, R.: Measurable selections of extrema. Ann Stat 1, 902-912 (1973)

Carmona, G.: Understanding some recent existence results for discontinuous games. Econ Theory (to appear)

Dasgupta, P., Maskin, E.: The existence of equilibrium in discontinuous economic games I: theory. Rev Econ Stud 53, 1-26 (1986)

Dasgupta, P., Maskin, E.: The existence of equilibrium in discontinuous economic games II: applications. Rev Econ Stud 53, 28-42 (1986) 
Glicksberg, I.: A further generalization of Kakutani's fixed point theorem with applications to Nash equilibrium points. Proc Natl Acad Sci USA 38, 170-172 (1952)

Hotelling, H.: The stability of economic competition. Econ J 39, 41-57 (1929)

Jackson, M.O., Simon, L.K., Swinkels, J.M., Zame, W.R.: Communication and equilibrium in discontinuous games of incomplete information. Econometrica 70, 1711-1740 (2002)

Lebrun, B.: Existence of an equilibrium in first price auctions. Econ Theory 7, 421-443 (1996)

Moore, J.C.: Mathematical Methods for Economic Theory 2. New York: Springer (1999)

Myerson, R.: Game Theory. Cambridge: Harvard University Press (1991)

Neveu, J.: Mathematical Foundations of the Calculus of Probability. San Francisco: Holden-Day (1965)

Pfanzagl, J.: Convexity and conditional expectations. Ann Probab 2, 490-494 (1974)

Reny, P.: On the existence of pure and mixed strategy Nash equilibria in discontinuous games. Econometrica 67, 1029-1056 (1999)

Simon, L.K.: Games with discontinuous payoffs. Rev Econ Stud 54, 569-597 (1987)

Simon, L.K., Zame, W.R.: Discontinuous games and endogenous sharing rules. Econometrica 58, 861-872 (1990)

Valadier, M.: Désintégration d'une mesure sur un produit. C.R Acad Sci Paris 267, 33-35 (1973) 